\title{
Deep Neural Network for Heart Disease Medical Prescription Expert System
}

\author{
Majzoob K. Omer ${ }^{1}$, Osama E. Sheta ${ }^{2}$, Mohamed S. Adrees ${ }^{3}$, Deris Stiawan ${ }^{4}$, Munawar A. Riyadi ${ }^{5}$, \\ Rahmat Budiarto ${ }^{6}$ \\ ${ }^{1}$ Faculty of Comp. Sc. \& I.T., AL Neelain University, Khartoum, Sudan \\ ${ }^{2,3,6}$ Faculty of Science, Zagazig University, Zagazig, Egypt \\ ${ }^{2}$ College of Comp. Sc. \& I.T., Albaha University, Albaha, Saudi Arabia \\ ${ }^{4}$ Faculty of Computer Science, Sriwijaya University, Indralaya, Indonesia \\ ${ }^{4,5}$ Faculty of Engineering, Universitas Diponegoro, Semarang, Indonesia
}

Article Info

Article history:

Received Apr 19, 2018

Revised Apr 27, 2018

Accepted May 2, 2018

\section{Keyword:}

Deep learning

Ischaemic heart disease

Neural networks

\begin{abstract}
One of the most common causes of death is Ischaemic heart disease (IHD). Clinical decisions are often made based on doctors' intuition and experience rather than on the knowledge-rich data hidden in the database, which leads to unwanted errors and excessive medical costs that affects the quality of service provided to patients. On the other hand, there is lack of cardiologist and IHD specialist in developing countries. Therefore, the development of an expert system that improves the diagnostic and therapeutic decision model of IHD creates a universal need. The expert system is developed based on the cardiologist expertises in diagnosing IHD symtomps and the given prescriptions. This work attempts to increase the accuracy and the effectiveness of the expert system to treat IHD patient by leveraging deep neural networks and adopting deep learning strategy for Retristic Boltzman Machine (RBM). The deep neural network in this work has 152 neurons in the input layer, 52 neurons in the output layer, and 4 hidden layer. Experimental results show that the proposed system achieves up to 0.00974 error level in the training sessions and average improvement of $0.7322 \%$ in term of accuracy compared to expert system with standard machine learning in the testing phase. Some results that have discrepancies are consulted to the cardiologist to confirm the results.
\end{abstract}

Copyright $@ 2018$ Institute of Advanced Engineering and Science. All rights reserved.

\section{Corresponding Author:}

Majzoob K. Omer

Faculty of Comp. Sc. \& I.T.,

AL Neelain University, Khartoum, Sudan.

E-mail: majzoob10@gmail.com

\section{INTRODUCTION}

Ischaemic heart disease (IHD) is related to various cardiovascular circumstances or risk factors and consequently, treatment can be complex. Thus, "treatment deserves a comprehensive management approach, including pharmacotherapeutic and invasive or surgical therapies, professional lifestyle interventions based on behavioural models of change, with different strategies from more basic, family-based to more structured and complex modalities, depending on the cardiovascular risk assessment and on concomitant diseases" [1]. Risk factor management concentrating on monitoring related cardiovascular risk factors, covering psychosocial support, physical activity advice and appropriate prescription of an adherence to cardioprotective drugs are integral to serving patients recover as normal a life as possible and advance their quality of life.

IHD is one of the most common causes of death in the world. This is the reason why the development of a broadly accepted expert system for accurately performing diagnostic and therapeutic 
decision model of ischaemic heart disease constitutes a general need. In addition, insufficient IHD specialist in developing countries especially in rural area requires a support of an expert system to help non-specialist to accurately diagnose and treat IHD patients.

Physicians need a systemic approach to actual clinical problems. Such a strategy ensures the maximum diagnostic accuracy at minimum risk and expense to the patient. Most cardiologists pursue a line of questioning to establish whether the actual chest pain is typically cardiac, a typical or non-cardiac in origin. It is therefore important to determine the sequence of the steps in the diagnosis of IHD. Clinical decisions are often made based on doctors' intuition and experience rather than on the knowledge-rich data hidden in the database. This practice leads to unwanted biases, errors and excessive medical costs which affects the quality of service provided to patients.

Many works (such as [2-6]) have already been carried out on the development of expert systems for IHD diagnosis and treatement with the use of intelligent systems such as fuzzy logic, genetic algorithm and artificial neural network. The existing expert systems using conventional neural network face problem with accuracy. This work attempts to develop an intelligent engine to improve the accuracy of such expert system by leveraging the deep learning concept. The expert system is expected to help cardiologists as well as general physicians (in case the absence of the cardiologists in rural area) to accurately treat the IHD patients.

\section{RESEARCH METHOD} input data.

This work uses the deep neural networks due to its advantace in processing inter-related string/text

\subsection{Dataset}

305 Patient clinical data related to IHD are collected from a hospital in Jakarta, Indonesia. The data is collected as symtomps and medicine prescriptions. Each patient information has ten attributes:

- Previous Disease Record (P1)

- $\quad$ Present Disease Record (P2)

- $\quad$ Personal Habit Record (P3)

- $\quad$ Physical Examination Results (P4)

- Cardio Vascular System (CVS)

- $\quad$ Respiratory System (RS)

- $\quad$ Per Abdomen (PA)

- $\quad$ Central Nervous system (CNS)

- Electrocardiogram (ECG)

- $\quad$ Blood Investigation (BI)

The details of the patient data/information are shown in Table 1 to Table 10

\begin{tabular}{cl} 
Table 1. & Physical Examination Results (P4) \\
\hline Code & \multicolumn{1}{c}{ Disease } \\
\hline 1 & Altered consciousness \\
2 & Orientation \\
3 & Dyspnlow pulse ea \\
4 & Fever \\
5 & Low pulse rate \\
6 & Normal pulse rate \\
7 & High pulse rate \\
9 & Low systolic blood pressure \\
9 & Normal blood pressure \\
10 & High blood pressure \\
11 & Low respiratory rate \\
12 & Normal respiratory rate \\
14 & High respiratory rate \\
15 & Pallor \\
16 & Jaundice \\
17 & Pedema feet \\
18 & Saturation less than $90 \%$ \\
19 & No abnormality detected (NAD) \\
20 & Restlessness \\
21 & Cyanosis \\
22 & Irregular pulse \\
23 & General condition - not satisfactory \\
24 & Facial puffiness \\
25 & Cold extremeties \\
\hline &
\end{tabular}

Table 2. Previous Disease Record (P1)

\begin{tabular}{cl}
\hline Code & \multicolumn{1}{c}{ Disease } \\
\hline 1 & Hypertension \\
2 & Diabetes Mellitus \\
3 & TB \\
4 & Bronchial Asthma \\
5 & Hyperthyroidism \\
6 & Hypothyrodism \\
7 & Old IHD \\
8 & Nil \\
9 & Interstitial Lung Disease (ILD) \\
10 & Cerebrovascular Accident (CVA) \\
11 & Rheumatoid arthritis \\
12 & Haemorrhoids (Bledding piles) \\
13 & Rheumaticd heart disease \\
14 & Atrial Fibrilation \\
15 & Chronic Obstructive Pulmonary Disease \\
16 & Supra Ventricular Techycardia (SVT) \\
17 & Febroid with Menorrhagia \\
18 & Dilated Cardiomyopathy \\
\hline
\end{tabular}


Table 3. Present Disease Record (P2)

\begin{tabular}{cl}
\hline Code & \multicolumn{1}{c}{ Disease } \\
\hline 1 & Chest pain \\
2 & Retrosternal pain \\
3 & Palpitations \\
4 & Breathlessness \\
5 & Sweating \\
6 & Persipiration \\
7 & Giddiness \\
8 & Nausea/ Vomitting \\
9 & Epigastric pain \\
10 & Left arm pain \\
11 & Syncope \\
12 & Unconsciousness \\
13 & Uneasiness/restlessness \\
14 & Back pain \\
15 & Heaviness in chest \\
16 & Difficulty in breathing \\
17 & Cough \\
18 & Swelling over feet \\
19 & Convulsion \\
20 & Headache \\
21 & Heartburn \\
22 & Jaw/throat pain \\
23 & Decrease apetite \\
24 & Epistaxis \\
25 & Haemoptysis \\
26 & Both shoulder pain \\
27 & Bleeding per rectum (PR) \\
28 & Haemetemesis \\
29 & Loose motion \\
\hline &
\end{tabular}

Table 5. Cardio Vascular System

\begin{tabular}{cl}
\hline Code & \\
\hline 1 & Hearth sounds \\
2 & Normal heart rate \\
3 & Tachycardia \\
4 & Bradycardia \\
5 & Regular heart rhythm \\
6 & Irregular heart rhythm \\
7 & Gallop sound \\
8 & No abnormality detected (NAD) \\
\hline
\end{tabular}

Table 7. Central Nervous System (CNS)

\begin{tabular}{|c|c|}
\hline Code & Findings \\
\hline 1 & Consciousness \\
\hline 2 & Orientation \\
\hline 3 & Focal deficit \\
\hline 4 & Restlessness \\
\hline 5 & No abnormality detected (NAD) \\
\hline
\end{tabular}

Table 4. Personal Habit Record (P3)

\begin{tabular}{cc}
\hline Code & Disease \\
\hline 1 & Smoking \\
2 & Tobacco \\
3 & Alcohol \\
4 & Nil \\
\hline
\end{tabular}

Table 6. Respiratory System

\begin{tabular}{cl}
\hline Code & \multicolumn{1}{c}{ Findings } \\
\hline 1 & Breath sounds preserved \\
2 & Breath sounds reduced \\
3 & Basal crepts \\
4 & Ronchi \\
5 & No abnormality detected (NAD) \\
\hline
\end{tabular}

Table 8. Per Abdomen (PA)

\begin{tabular}{cl}
\hline Code & \multicolumn{1}{c}{ Findings } \\
\hline 1 & Liver (hepatomegaly) \\
2 & Spleen (splenomegaly) \\
3 & Free fluid present \\
4 & Abdominal distension \\
5 & Obesity \\
6 & No abnormality detected (NAD) \\
\hline
\end{tabular}


Table 9. ECG

\begin{tabular}{cc}
\hline Code & Disease \\
\hline 1 & ST elevation \\
2 & Anterior wall \\
3 & Anteroseptal \\
4 & Inferior \\
5 & Inferoposterior \\
6 & Lateral \\
7 & Septal \\
8 & High lateral \\
9 & T wafe inversion \\
10 & ST depression \\
11 & QS complex \\
12 & LBBB \\
13 & Inferior and lateral (4\&6) \\
14 & Atrial fibrillation \\
15 & RBBB \\
16 & VBP's \\
17 & Sinus tachycardia \\
18 & Sinus rhythm \\
19 & Supraventricular tachycardia SVT \\
20 & Left ventricular hypertrophy (LVH) \\
21 & Ventricular tachycardia/ fibrillation \\
\hline
\end{tabular}

Table 10. Blood Investigation (BI)

\begin{tabular}{cc}
\hline Code & Disease \\
\hline 1 & Cardiac enzymes (High) \\
2 & Blood sugar test normal \\
3 & Blood sugar test low \\
4 & Blood sugar test high \\
5 & Kidney function test deranged \\
6 & Lipid profile normal \\
7 & Lipid profile abnormal \\
8 & Complete blood count normal \\
9 & Leukocytosis \\
10 & Anemia \\
11 & Thrombocytopenia \\
12 & Urine routine \\
13 & Troponin T/I (positive) \\
14 & No abnormality detected (NAD) \\
15 & Chest X-ray (cardiomegaly) \\
16 & 2-D-Echo RWMA \\
17 & 2-D-Echo LV CLOT \\
18 & 2-D-Echo LVH \\
19 & Hypokalemia \\
20 & Hyponatremia \\
21 & Thyroid profile (abnormal) \\
22 & 2D-Echo - poor LVEF \\
23 & Hypercalemia \\
24 & \\
\hline &
\end{tabular}

The medicines as treatments for the IHD along with their codes (MIDs) are shown in Table 11. While Table 12 and Table 13 show the twenty of clinical data examples on symptoms examinations, and the medicines prescribed by the physician, respectively.

Table 11. The Medicines and Their Codes

\begin{tabular}{|c|c|c|c|}
\hline MID & Medicine name & MID & Medicine name \\
\hline 1 & Alprazolam & 27 & Fortwin Inj \\
\hline 2 & Amlodepine & 28 & Diazepam Inj \\
\hline 3 & Aspirin & 29 & Nitroglycerin Inj \\
\hline 4 & Atenolol & 30 & Ciprofloxacin Inj \\
\hline 5 & Atorvastatin & 31 & Cardarone \\
\hline 6 & Clopidogrel & 32 & Dobutamine Inj \\
\hline 7 & DIGOXIN & 33 & Levolin (Nebolize) \\
\hline 8 & Diltiazem & 34 & Methyl prednisolone \\
\hline 9 & Diphenylhydantoin Sodium & 35 & Oral Hypoglycaemics \\
\hline 10 & Enalapril & 36 & Lactulose syrup \\
\hline 11 & Furosemide & 37 & Eltoxin \\
\hline 12 & Ethamsylate & 38 & Dextrose $25 \%$ Inj \\
\hline 13 & Insulin Inj & 39 & Dopamine \\
\hline 14 & Iso sorbide dinitrate & 40 & Warfarin sodium \\
\hline 15 & Losartan & 41 & Iron Capsule \\
\hline 16 & Metoprolol & 42 & Neomercazole \\
\hline 17 & Nikorandil & 43 & Betahistine HCL \\
\hline 18 & Ondansetron & 44 & Kesol Syrup / Inj \\
\hline 19 & Paracetamol & 45 & Vit -B12 \\
\hline 20 & Perindopril & 46 & Ethamysylate \\
\hline 21 & Ramipril & 47 & Fen ofibrate \\
\hline 22 & Trimetazidine & 48 & Inj Calcium Gluconate \\
\hline 23 & Streptokinase Inj & 49 & (Glucose + Insulin ) Drip \\
\hline 24 & Taxim Inj & 50 & K-bind powder \\
\hline 25 & Enoxaparin Inj & 51 & Syp.Gellusil / Mucaine gel \\
\hline 26 & Rabeprazole Inj & 52 & Sucralfate Syp \\
\hline
\end{tabular}


Table 12. Examples of Symptoms Examinations of 20 Patients

\begin{tabular}{cccccccccc}
\hline P1 & P2 & P3 & P4 & CVS & RS & PA & CNS & ECG & BI \\
\hline 1 & 15,16 & 4 & $6,9,12$ & 8 & 4 & 6 & 4 & 10 & 2 \\
1,2 & 4,5 & 4 & $1,2,7,13,14,16$ & 3,5 & 3 & 5 & 4 & 12 & 4 \\
8 & $13,7,5$ & 4 & $1,6,12$ & 8 & 4 & 5 & 4 & 9 & 14 \\
2 & $1,2,8$ & 2 & $7,8,13,14$ & 8 & 4 & 5 & 4 & 2 & 7 \\
2 & $1,2,13,5$ & 4 & 7,10 & 8 & 4 & 5 & 4 & 1,3 & 14 \\
8 & 1,4 & 1 & 6,10 & 8 & 4 & 5 & 4 & 3 & 2 \\
1 & $15,3,4$ & 4 & $6,10,12$ & 8 & 4 & 5 & 4 & 9 & 14 \\
1,7 & 1,17 & 4 & 6,9 & 6 & 4 & 5 & 4 & 9 & 9 \\
7 & 18 & 4 & $3,7,9,16$ & 3 & 3 & 1 & 4 & 11,3 & 14 \\
$9,2,3$ & 4 & 4 & $7,9,13,18$ & 3 & 3,5 & 5 & 4 & 11,3 & $9,10,4$ \\
8 & 1,5 & 4 & 6,10 & 8 & 4 & 5 & 4 & 3 & $9,1,2$ \\
8 & 1 & 1 & 19 & 8 & 4 & 5 & 4 & 1,9 & 14,2 \\
$1,2,6$ & $1,5,8$ & 4 & $6,10,12,14$ & 8 & 4 & 5 & 4 & 1,2 & $1,4,9,10$ \\
1,7 & $4,3,5,6,15$ & 4 & $3,7,10,13,17,18$ & 3 & 3,5 & 5 & 4 & 4,11 & 15 \\
7,10 & 19 & 2 & $6,9,14$ & 8 & 4 & 5 & 3 & 11,4 & 14 \\
1 & 1 & 4 & $6,9,12$ & 8 & 4 & 5 & 4 & 10,9 & 14 \\
$1,2,7$ & $3,5,6,7$ & 4 & $6,9,12$ & 8 & 4 & 5 & 4 & 9 & 3 \\
7 & 1 & 4 & $6,9,12$ & 8 & 4 & 5 & 4 & 11,4 & 14 \\
4 & $2,5,6,13$ & 4 & $6,10,12,17,20$ & 8 & 4 & 5 & 5 & 1,4 & $4,9,16,17$ \\
7 & $1,5,6,13$ & 4 & $20,17,6,10,13$ & 8 & 4 & 5 & 5 & $1,3,11,4$ & $2,9,10,16,17$ \\
\hline
\end{tabular}

Table 13. The Corresponding Medicine Prescriptions for the 20 Patients

\begin{tabular}{|c|c|c|c|c|c|c|c|c|c|c|c|c|c|c|c|c|}
\hline $\begin{array}{c}\text { MD } \\
1\end{array}$ & $\begin{array}{c}\text { MD } \\
2\end{array}$ & $\begin{array}{c}\text { MD } \\
3\end{array}$ & $\begin{array}{c}\mathrm{MD} \\
4\end{array}$ & $\begin{array}{c}\text { MD } \\
5\end{array}$ & $\begin{array}{c}\text { MD } \\
6\end{array}$ & $\begin{array}{c}\text { MD } \\
7\end{array}$ & $\begin{array}{c}\text { MD } \\
8\end{array}$ & $\begin{array}{c}\text { MD } \\
9\end{array}$ & $\begin{array}{c}\text { MD } \\
10\end{array}$ & $\begin{array}{c}\text { MD } \\
11\end{array}$ & $\begin{array}{c}\text { MD } \\
12\end{array}$ & $\begin{array}{c}\text { MD } \\
13\end{array}$ & $\begin{array}{c}\text { MD } \\
14\end{array}$ & $\begin{array}{c}\text { MD } \\
15\end{array}$ & $\begin{array}{c}\text { MD } \\
16\end{array}$ & MD 17 \\
\hline 11 & 13 & 14 & 17 & 3 & 5 & 7 & 10 & 29 & 19 & 30 & & & & & & \\
\hline 14 & 6 & 5 & 1 & 25 & & & & & & & & & & & & \\
\hline 1 & 3 & 5 & 6 & 13 & 14 & 17 & 19 & 21 & 23 & 25 & 26 & 27 & 29 & 36 & & \\
\hline 1 & 3 & 5 & 6 & 14 & 17 & 19 & 21 & 23 & 25 & 26 & 27 & 29 & 36 & & & \\
\hline 3 & 14 & 19 & 15 & & & & & & & & & & & & & \\
\hline 25 & 6 & 3 & 29 & 5 & 17 & 1 & 21 & 16 & & & & & & & & \\
\hline 14 & 3 & 5 & 6 & 1 & 26 & & & & & & & & & & & \\
\hline 29 & 1 & 3 & 5 & 25 & 17 & 16 & 27 & 24 & & & & & & & & \\
\hline 32 & 11 & 20 & 33 & 14 & & & & & & & & & & & & \\
\hline 14 & 11 & 34 & 3 & 5 & 33 & 35 & 24 & & & & & & & & & \\
\hline 23 & 1 & 5 & 6 & 29 & 25 & 17 & 31 & 16 & 21 & 36 & & & & & & \\
\hline 25 & 1 & 3 & 5 & 29 & 17 & 16 & & & & & & & & & & \\
\hline 23 & 25 & 26 & 27 & 28 & 29 & 13 & 1 & 3 & 5 & 16 & 17 & 18 & 21 & 37 & 6 & 36 \\
\hline 11 & 21 & 29 & 1 & 3 & 5 & 17 & 7 & 28 & 19 & 15 & & & & & & \\
\hline 9 & 30 & 33 & 3 & 19 & & & & & & & & & & & & \\
\hline 14 & 1 & 3 & 5 & 2 & & & & & & & & & & & & \\
\hline 38 & 3 & 14 & 5 & 1 & & & & & & & & & & & & \\
\hline 25 & 2 & 3 & 5 & 6 & 29 & 17 & 16 & & & & & & & & & \\
\hline 2 & 3 & 5 & 6 & 13 & 14 & 17 & 19 & 21 & 23 & 25 & 26 & 27 & 28 & 29 & 36 & \\
\hline 2 & 3 & 5 & 6 & 14 & 16 & 17 & 19 & 21 & 25 & 26 & 27 & 28 & 29 & 36 & & \\
\hline
\end{tabular}

\subsection{Deep neural network}

Deep Neural Learning or Deep Neural Network (DNN) is a subset of machine learning in Artificial Intelligence (AI) that has networks which are capable of learning unsupervised from data that is unstructured or unlabeled. The deep neural network consists of more than 1 layer in its hidden layer [7-10]. The DNN in this work, has 152 neurons in the input layer, 52 neurons in the output layer, and 4 layers in the hidden layer. Each attribute of patient is represented by binary numbers. The number of digit representing the attribute depends on the number of fields shown in Table 1 to Table 10 . The number of digit of $\mathrm{P} 1=18, \mathrm{P} 2=29, \mathrm{P} 3=4$, $\mathrm{P} 4=25, \mathrm{CVS}=8, \mathrm{RS}=5, \mathrm{PA}=6, \mathrm{CNS}=5, \mathrm{ECG}=21, \mathrm{BI}=24$. Thus, the total is 152 as the number of neurons for the input. Digit 1 represents that the symptom exists, otherwise is 0 . For example, the input for the fourth patient record in Table 12 is as follows. 011011101000000000000000011001000000010000000000000000 0001.

0000001001000000000000000000000010001000001000010

101000000000000000000000000000000010000000000 . The output is medicine prescriptions as shown in Table 11. So, there are 52 neurons in the output layer. As illustration, the fourth patient's medicines prescription in Table 13 is represented as: 
1010110000000100101010101110100000010000000000000000. Figure 1 illustrates the architecture of the DNN.

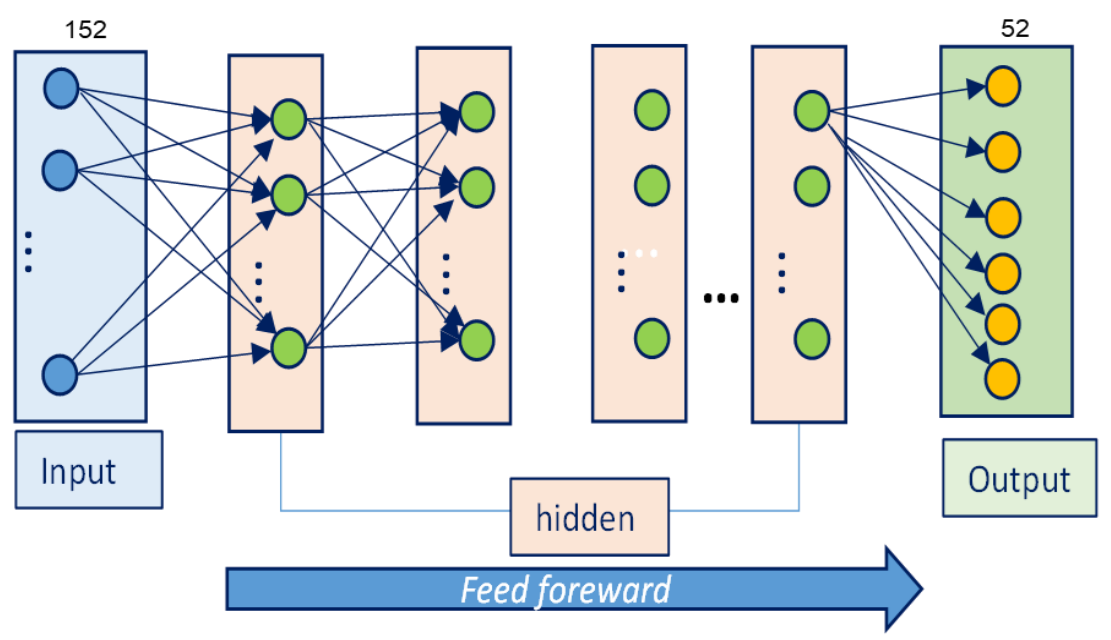

Figure 1. Architecture of the DNN

\subsection{The Algorithm}

This work adopts the deep learning method for Retristic Boltzman Machine (RBM) from the works in [6] and [7]. 250 patient's data are used for training and the other 55 data are for testing. The main aim of the RBM training algorithm is to maximize the product of probabilities assigned to some training set $\mathrm{V}$ (a matrix, each row of which is treated as a visible vector $\mathrm{v}$ ):

$$
\underset{\mathrm{w}}{\arg \max } \prod_{v \in V} P(v)
$$

The algorithm optimizes the weight matrix W [6]. The algorithm performs Gibbs sampling and is used inside a gradient descent procedure (similar to the way backpropagation is used inside such a procedure when training feedforward neural networks) to compute weight update. The basic, single-step contrastive divergence procedure for a single sample is summarized as follows:

a. Take a training sample $v$, compute the probabilities of the hidden units and sample a hidden activation vector $h$ from the probability distribution.

b. Compute the outer product of $v$ and $h$ and call this the positive gradient.

c. From $h$, sample a reconstruction $v^{\prime}$ of the visible units, then resample the hidden activations $h^{\prime}$ from this. (Gibbs sampling step)

d. Compute the outer product of $v^{\prime}$ and $h^{\prime}$ and call this the negative gradient.

e. Let the update to the weight matrix $\boldsymbol{W}$ be the positive gradient minus the negative gradient, times some learning rate:

$$
\Delta W=\in\left(v h^{T}-v^{\prime} h^{\prime T}\right)
$$

f. Update the biases $a$ and $b$ analogously:

$$
\begin{aligned}
& \Delta \mathrm{a}=\in\left(\mathrm{v}-\mathrm{v}^{\prime}\right) \Delta\left\{\text { \displaystyle } \backslash \text { Delta } \mathrm{b}=\text { lepsilon }\left(\mathrm{h}-\mathrm{h}^{\prime}\right)\right\} \\
& \Delta \mathrm{a}=\in\left(\mathrm{h}-\mathrm{h}^{\prime}\right)
\end{aligned}
$$

\subsection{Implementation}

The algorithm is implemented using a high-end PC machine with the following specifications: Processor name: Intel Core i7-7700, Processor speed 3.8 GHz, RAM 16 GB, Storage 2.5 TB and Windows $10 \mathrm{O} / \mathrm{S}$. Tensorflow utility/library is used on the Phyton platform. 


\section{RESULTS AND ANALYSIS}

\subsection{Training Results}

Five (5) series of training were conducted with the same number of epochs. Figure 2 shows the training results. The average error is 0.009895 . The training time was also recorded and in average the proposed system achieved a rational training time compared to the standard/conventional neural network. It was $15 \%$ slower.

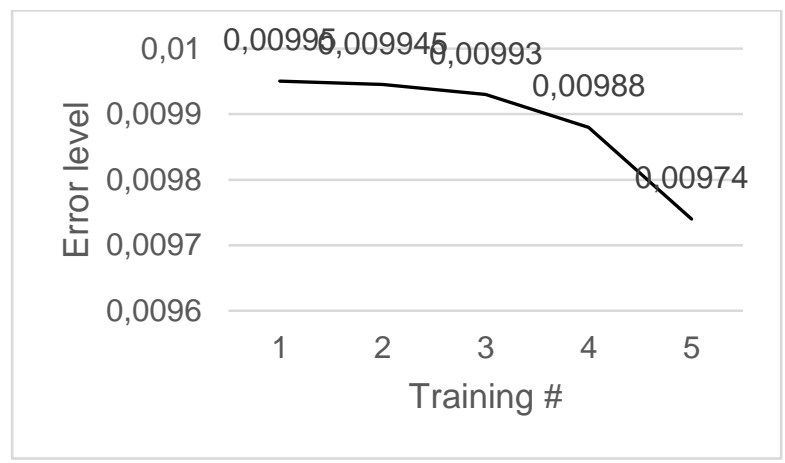

Figure 2. Training results

\subsection{Testing Results}

Fifty-five (55) data testing were used and some data that used during the testing. We compared the accuracy of the proposed expert system (using DNN) with standard/conventional Neural Network (NN). Five experiments are carried out and the results are shown in Figure 3. The average accuracy of the proposed expert system with DNN is: $(99.60+99.75+99.801+99.801+99.851) / 5=99.7874 \%$, whereas the average accuracy of the expert system with $\mathrm{NN}$ is: $(99.16+99.002+99.1+99.002+99.012)=99.0552$. Thus, the proposed expert system with DNN improves the accuracy with $0.7322 \%$.

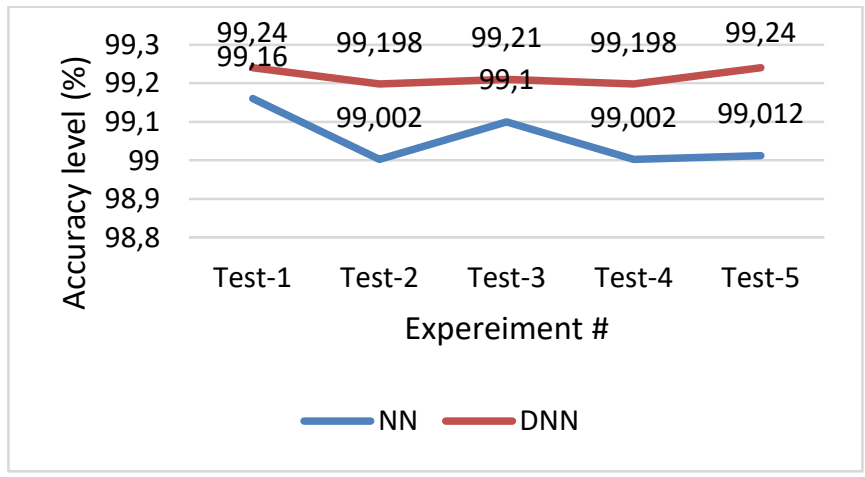

Figure 3. Accuracy of the proposed system

Table 14 shows the sample results of the testing that have discrepancies compared with the actual medicine prescriptions given by the physician. Analysis from the physician are given for some discrepancies of the results. For example, for patient number 4:

- Medicine no. 16 is suggested by the system as additional prescription. It is beneficial as it reduces the heart rate and thereby reduces workload and improves outcome.

- Medicine no. 19 is not given by the system. It is an antipyretic drug (to reduce fever) or an analgesic that if it is given, will not affect the cardiac outcome.

- $\quad$ Medicine No. 28 is injectable form of medicine no. 14 which the system has already prescribed.

- Medicine no. 36 is not given by the system. It is a laxative (Stool Softener) is given to patients who complain passing hard stools which cannot be judged by the system. 
Table 14. Some Testing Results

\begin{tabular}{|c|c|c|c|c|}
\hline $\begin{array}{c}\text { Patient } \\
\#\end{array}$ & Ssytem's Prescription & Physician Prescription & Not prescribed & $\begin{array}{l}\text { Additional } \\
\text { prescription }\end{array}$ \\
\hline 1 & $1,3,5,6,14,25$ & $3,5,7,10,11,13,14,17,19,29,30$ & $7,10,11,13,14,17,19,30$ & 1,6 \\
\hline 2 & $1,3,5,6,14,25$ & $1,5,6,14,25$ & Nil & 3 \\
\hline 3 & $1,3,5,6,14,25$ & $\begin{array}{c}1,3,5,6,13,14,17,19,21 \\
3,25,26,27,29,36\end{array}$ & $13,17,19,21,23,25,26,27,29,36$ & Nil \\
\hline 4 & $\begin{array}{c}1,3,5,6,14,16,17,21,23,25 \\
, 26,27,28\end{array}$ & $1,3,5,6,14,17,19,21,23,25,26,27,29,36$ & $19,29,36$ & 16 \\
\hline 5 & $1,3,5,6,14,25$ & $3,14,15,19$ & 15,19 & $1,5,6,25$ \\
\hline 6 & $1,3,5,6,14,25$ & $3,5,11,14,24,33,34,35$ & $11,24,33,34,35$ & $1,6,25$ \\
\hline 7 & $1,3,5,6,14,25$ & $1,3,5,6,14,26$ & 26 & 25 \\
\hline 8 & $1,3,5,6,14,25$ & $1,3,5,16,17,24,25,27,29$ & $16,17,24,27,29$ & 14 \\
\hline 9 & $1,3,5,6,14,25$ & $11,14,20,32,33$ & $11,20,32,33$ & $1,3,5,6,25$ \\
\hline 10 & $1,3,5,6,14,25$ & $1,3,5,6,16,17,21,25,29$ & $16,17,21,29$ & Nil \\
\hline
\end{tabular}

\section{CONCLUSION}

A deep neural network has been used to increase the accuracy of the expert system for IHD treatment. The proposed expert system that uses deep neural network has improved the accuracy by $0.7322 \%$ compared to the expert system that uses conventional neural network. in some cases, the system suggested new prescriptions for some patients, however, those prescriptions do not affect the cardiatic problems, according the advices from the physician. On the other hand, the medicines that were prescribed by the physician but were not prescribed by the system do not significantly affect the IHD. In other word, it increases efficiency in term of cost for IHD treatment.

\section{REFERENCES}

[1] Piepoli MF, Hoes AW, Agewall S, Albus C, Brotons C, Catapano AL, Cooney M-T, Corra` U, Cosyns B, Deaton C, Graham I, Hall MS, Hobbs FDR, Løchen M-L, Lollgen H, Marques-Vidal P, Perk J, Prescott E, Redon J, Richter DJ, Sattar N, Smulders Y, Tiberi M, van derWorp HB, van Dis I, Verschuren WMM. 2016 European Guidelines on cardiovascular disease prevention in clinical practice. Eur Heart J 2016; doi:10.1093/eurheartj/ehw106.

[2] Kékes E, Kakas M, Aszalos J, Preda I, Barcsak J, Kovacs J, and Antaloczy Z. An Expert System for the Diagnosis and Treatment of Ischaemic Heart Disease: Cardexp. In: Carson E.R., Kneppo P., Krekule I. (eds) Advances in Biomedical Measurement, 1988, Springer, Boston, MA.

[3] Alqudah, A.M. Fuzzy expert system for coronary heart disease diagnosis in Jordan, Health and Technology. 2017:7(2-3): 215-222.

[4] Muthukaruppan S, Er MJ. A hybrid particle swarm optimization based fuzzy expert system for the diagnosis of coronary artery disease. Expert Syst Appl. 2012;39(14):11657-65.

[5] Hassan N, Sayed OR, Khalil AM, Ghany MA. Fuzzy Soft Expert System in Prediction of Coronary Artery Disease. International Journal of Fuzzy Systems. 2017 Oct 1;19(5):1546-1559. Available from, DOI: 10.1007/s40815-016-0255-0.

[6] Adeli A, Neshat M, A Fuzzy Expert System for Heart Disease Diagnosis, International Conference of Computer Engineers and Scientist, vol. 1, Hongkong, March 2010.

[7] Hinton, G.E., Osindero, S., \& Teh, Y.-W. A fast learning algorithm for deep belief nets. Neural Computation, 20061(8): 1527-1554.

[8] Salakhutdinov R, Hinton, G.E. Deep Boltzmann Machines 12th International Confe-rence on Artificial Intelligence and Statistics (AISTATS) 2009, Clearwater Beach, Florida, USA. Volume 5 of JMLR: W\&CP 5.

[9] Lopes N and Ribeiro B. Machine Learning for Adaptive Many-Core Machines - 155 A Practical Approach, Studies in Big Data 7, DOI: 10.1007/978-3-319-06938-8_8, c Springer International P Publishing Switzerland, 2015. Abdel-Zaher A.M, Eldeib A.M. Breast cancer classification using deep belief, Expert Systems With Applications

[10] Abdel-Zaher A.M, Eldeib A.M. Breast cancer classification using deep belief, Expert Systems With Applications 46 (2016):139-144. 